\title{
Los batallones de Acción Integral y la construcción de paz en Colombia
}

\section{The Integral Action battalions and the construction of peace in Colombia Os batalhões de Ação Integral e a construção da paz na Colômbia}

\author{
María Eugenia Bonilla Ovallos ${ }^{a^{*}} \mid$ Ana María Villada Gómez \\ ${ }^{a}$ https://orcid.org/0000-0002-9456-0062 \\ ${ }^{\text {b }}$ https://orcid.org/0000-0002-763I-1767 \\ Universidad Autónoma de Bucaramanga, Bucaramanga, Colombia
}

- Fecha de recepción: 2019-12-13

- Fecha concepto de evaluación: 2020-05-20

- Fecha de aprobación: 2020-05-27 https://doi.org//0.22335/rlct.v/2i2.I I 25

\begin{abstract}
Para citar este artículo / To reference this article / Para citar este artigo: Bonilla Ovallos, M. E., \&Villada Gómez, A. M. (2020). Los batallones de Acción Integral y la construcción de paz en Colombia. Revista Logos Ciencia \& Tecnología, 12(2), 70-83. https://doi.org/10.22335/ rlct.v12i2.1 I 25
\end{abstract}

\section{RESUMEN}

Tras la firma del Acuerdo de Paz entre el gobierno de Colombia y las FARC, la estrategia no armada del Ejército se configuró como clave en el posacuerdo. Este artículo indaga sobre las debilidades, las oportunidades, las fortalezas y las amenazas que enfrentan los batallones de Acción Integral del Ejército Nacional de Colombia en los departamentos de Santander y Cundinamarca. Para esto se realizó una revisión documental, se encuestaron oficiales, suboficiales, soldados y civiles que integran estas unidades, y se entrevistó a los comandantes de estos batallones en ambos departamentos. Los resultados apuntan a ratificar la importancia de la Acción Integral del Ejército en la construcción de paz, la necesidad de fortalecer la articulación interinstitucional frente a las acciones no armadas y de avanzar hacia la solidez presupuestal, técnica, logística y profesionalizante de estos batallones.

Palabras clave: Ejército, acción integral, paz, posacuerdo, desarrollo 


\begin{abstract}
After the signing of the Peace Agreement between the Colombian government and the FARC, the Army's unarmed strategy became the key in the post-agreement. This article investigates the weaknesses, opportunities, strengths and threats facing the Comprehensive Action battalions of the Colombian National Army in the departments of Santander and Cundinamarca. For this, a documentary review was carried out and officers, non-commissioned officers, soldiers and civilians that make up these units were surveyed, and the commanders of these battalions in both departments were interviewed. The results aim to ratify the importance of the Army's Comprehensive Action in the construction of peace, the need to strengthen the inter-institutional articulation against unarmed actions, and to advance towards the budgetary, technical, logistical and professional strength of these battalions.
\end{abstract}

Keywords: army, comprehensive action, peace, post-agreement, development

\title{
RESUMO
}

Após a assinatura do Acordo de Paz entre o governo colombiano e as FARC, a estratégia desarmada do Exército tornou-se a chave no pós-acordo. Este artigo investiga as fraquezas, oportunidades, pontos fortes e ameaças enfrentados pelos batalhões de Ação Integral do Exército Nacional da Colômbia nos departamentos de Santander e Cundinamarca. Para isso, foi realizada uma revisão documental, oficiais, suboficiais, soldados e civis que compõem essas unidades foram entrevistados e os comandantes desses batalhões em ambos departamentos. Os resultados apontam para ratificar a importância da Ação Integral do Exército na construção da paz, a necessidade de fortalecer a articulação interinstitucional contra ações desarmadas e avançar em direção à força orçamentária, técnica, logística e profissional desses batalhões.

Palavras-chave: Exército, ação abrangente, paz, pós-acordo, desenvolvimento

La firma del Acuerdo de Paz entre el gobierno de Juan Manuel Santos y las Fuerzas Armadas Revolucionarias (FARC), en noviembre del 2016, reconfiguró el papel del Ejército en el marco del posacuerdo (Guevara, Silvia \& Villegas, 2018). Este papel se soportó en la implementación de una doctrina militar denominada Plan Damasco, cuyo propósito ha sido la búsqueda de una paz estable y duradera, mediante la vinculación de miembros del Ejército con operaciones de paz y trabajo no armado. En consecuencia, en el 2017, se creó en Colombia el Comando de Apoyo de Acción Integral y Desarrollo del Ejército (CAAIDE), integrado por dos brigadas y ocho batallones, cuya orientación ha sido la estrategia comunitaria.

Este artículo analiza las oportunidades, las fortalezas, las amenazas y las debilidades que enfrentan los batallones de Apoyo de Acción Integral y Desarrollo (BAAID) en los departamentos de Santander y Cundinamarca, a fin de garantizar en el corto y mediano plazo estrategias no armadas que promuevan el desarrollo social en el marco del posacuerdo en Colombia. Con el propósito de alcanzar este objetivo se revisan las directrices nacionales que han contribuido a la misión no armada de los militares en Colombia entre 1953 y el 2018, se describen las características del CAAIDE y los BAAID en ambos departamentos y se analiza la percepción que estos tienen sobre las estrategias no armadas que buscan promover el desarrollo social en el marco del posacuerdo en Colombia.

La importancia de este artículo sobre una de las instituciones más herméticas del Estado se fundamenta en el grado de novedad, pues a la fecha no se han adelantado investigaciones académicas que se concentren en este objeto de investigación. Adicionalmente, los resultados permiten esbozar una serie de recomendaciones orientadas a potenciar la oportunidad que esta misión institucional representa para el mejoramiento de la imagen del Ejército, la construcción de paz y el desarrollo del país.

El artículo se estructura en cuatro apartados. En primer lugar, se presentan los pormenores del diseño metodológico implementado. Posteriormente, se exponen los resultados obtenidos y, por último, se presentan las conclusiones a la luz de los objetivos propuestos. . 


\section{Metodología}

Este artículo se soporta en una metodología de tipo mixta, dado que se emplearon técnicas de recolección de información tanto cuantitativas como cualitativas con el fin de alcanzar los objetivos propuestos (Pereira, 20II). Asimismo, se enmarca en los estudios de tipo exploratorio, en la medida en que no se han realizado previamente trabajos sobre los BAAID en Santander y Cundinamarca, debido a la complejidad de la estructura jerarquizada $y$ hermética del Ejército Nacional. Un estudio exploratorio es una pequeña versión de una investigación mayor que permite evidenciar cuestiones de orden metodológico, descubrir problemas y exponer la viabilidad de los instrumentos y las técnicas aplicadas (Muñoz, 20II).

Las técnicas de recolección de información utilizadas fueron varias. La primera fue la revisión documental. Esta técnica consiste en desarrollar rastreos de textos, documentos oficiales $y$ experiencias investigativas previas que hayan abordado el objeto de estudio (Guevara, 2016). En este sentido, se revisaron documentos que permitieron construir una línea de tiempo sobre las directrices nacionales que han contribuido a la misión no armada de los militares en Colombia durante los gobiernos que estuvieron vigentes entre 1953 y 2018 , así como a comprender la base legal, jurídica y política que dio origen a los BAAID.

La segunda técnica fue la producción de datos por medio de la aplicación de encuestas realizadas de manera online entre los meses de septiembre y octubre del 2019. Los cuestionarios se enviaron a los celulares de los integrantes de los BAAID de Santander y Cundinamarca (esto es, soldados, suboficiales, oficiales y civiles), quienes por medio de un enlace accedieron y diligenciaron la encuesta. En total se realizaron 72 encuestas, 49 en el batallón de Santander y 23 en el batallón de Cundinamarca (52\% y $20 \%$ de la población, respectivamente).

De manera complementaria, se realizaron, en septiembre del 2019, dos entrevistas semiestructuradas a los comandantes militares que lideraban la estrategia de Acción Integral en cada uno de los dos BAAID objeto de estudio. Finalmente, se solicitó de manera formal información a cada batallón sobre el personal vinculado a la unidad, el presupuesto anual asignado, la jurisdicción a cargo y la relación de programas, proyectos y acciones realizadas en el 2018. Esta información la brindó el BAAID de Santander en su totalidad, mientras que el BAAID de Cundinamarca la suministró de manera incompleta.

Las variables de análisis que se definieron en la investigación fueron tres: (I) Políticas nacionales sobre la misión no armada del Ejército; (2) Características del Comando de Apoyo de Acción Integral y Desarrollo del Ejército (CAAIDE);y (3) Características de los Batallones de Apoyo de Acción Integral y Desarrollo (BAAID). Para cada variable se plantearon dimensiones e indicadores, tal como puede apreciarse en la tabla I.

Tabla I. Variables, dimensiones e indicadores de análisis

\section{Variables}

Políticas nacionales sobre la misión no armada del Ejército

Características del Coman- Misión, objetivos, Estructura territorial. do de Apoyo de Acción

Integral y Desarrollo del Ejército (CAAIDE)

Características de los Características generales (jurisdicción, recursos humanos, batallones de Apoyo de recursos financieros líneas de intervención). Acción Integral y Desarrollo (BAAID)

\section{Dimensiones} 2018 Indicadores

Revisión documental

Número de departamentos a cargo.

Revisión documental Aspectos sociodemográficos (género, grupo etareo, tien
po de servicio, experiencia en labores operacionales).

Conocimiento sobre Acción Integral (nivel de conocimiento, formación recibida, experiencia en Acción Integral). Número de departamentos a cargo, número,
perfil y grado militar de los integrantes, presupuesto anual, actividades realizadas.

Hombre, mujer, género diverso. Edad. Años de permanencia en el Ejército Nacional, tiempo de vinculación en acciones armadas.

Alto, medio, bajo nivel de conocimiento. Cursos realizados en Acción Integral. Institución formadora. Años de experiencia en labores sociales y Acción Integral.

Percepción frente a la estrategias de Acción Integral Reconocimiento alto, medio, bajo. Satisfacción (nivel de reconocimiento recibido, grado de satisfacción alta, media, baja. Tipo de experiencias positivas. de su labor, experiencias positivas de la labor no armada, Razones por las que nos les gusta pertenecer a cuestionamientos sobre el funcionamiento de los BAAID, los BAAID. Acciones de mejora. propuesta de fortalecimiento de los BAAID). 
Resultados de la investigación

\section{Políticas nacionales sobre la misión}

\section{no armada del Ejército}

Los inicios de las acciones no armadas del Ejército se registran en Colombia desde la presidencia de Gustavo Rojas Pinilla (1953-1957), quien dio a los militares la potestad de asistir a las comunidades más vulnerables del país, mediante la promoción de acciones orientadas al fortalecimiento de la infraestructura rural (Suárez, 20I0).

Posteriormente, durante el gobierno de Alberto Lleras Camargo (1958-1962), se contempló la inversión social dentro de los planes de acción diseñados por el Ejército Nacional, destinando recursos a esta línea de intervención para el restablecimiento del orden público (CGFM, 2017). Los comandantes de los batallones comenzaron a interesarse en obtener el afecto de los campesinos de las zonas con presencia de guerrillas, implementando estrategias cívico-militares para acercarse a la comunidad (Valencia, 2006).

Estas estrategias se materializaron en la década de los sesenta con el Plan Lazo, el Plan Andes y el Plan Perla, los cuales acentuaron los objetivos de la acción no armada del Ejército en Colombia, enfatizando en el tratamiento social integral del conflicto (CGFM, 20I7).

La primera vez que el trabajo social del Ejército colombiano escaló al escenario social y político del Estado fue en el gobierno de Guillermo León Valencia (1962-1966), cuando el entonces Ministro de Defensa, General Alberto Ruiz Novoa, presentó en 1962 el Plan Lazo, enfocado en el esfuerzo no armado en beneficio de la población. La propuesta fue trabajar en conjunto con médicos, odontólogos e ingenieros en el propósito de atender demandas de salud de la comunidad, reconstruir viviendas y huertas, brindar servicios de peluquería y zapatería, entre otras acciones, con el fin de buscar la pacificación del país (CGFM, 20I7).

Luego, con el Plan Andes en 1963, se indicó la necesidad de integrar la acción cívico militar en todos los niveles del Gobierno nacional, con el objetivo de obtener resultados favorables en temas relacionados con el orden público. De esta manera, la estrategia se centró en mitigar los factores que pudieran incidir en el desarrollo de las regiones y así favorecer la intervención militar en zonas de conflicto (Cabrera, 2005).

Posteriormente, el presidente Carlos Lleras Restrepo (1966-1970) diseñó, en 1968, el Plan Perla, con el que se ordenó a las brigadas del Ejército informar sobre urgencias no militares en sus jurisdicciones. La información que debían suministrar estaba relacionada con vías, escuelas, puestos de salud, titulación de tierras y ordenamiento territorial. En general, el Plan Perla establecía, por parte del Ejército, un apoyo contundente frente a las necesidades específicas de cada región, orientando la intervención del Estado colombiano para impulsar el desarrollo (Valencia, 2006).

Durante el gobierno de Misael Pastrana Borrero (19791974) se creó, en 1973, el Comando General de las Fuerzas Militares el Departamento No. 5 de Estado Mayor Conjunto, con el objetivo de orientar una labor cooperativa entre las fuerzas que centralizara la ejecución de las relaciones cívico-militares (Silva, 2014).

Con la nueva Constitución Política de Colombia se creó, en 1991, la Escuela de Relaciones Civiles y Militares del Ejército (ERCM), la cual tuvo como finalidad formar a los miembros del Ejército, la Armada y la Fuerza Aérea como especialistas en operaciones psicológicas (CGFM, 2017).

En el gobierno de Álvaro Uribe Vélez (2002-2010) se estableció un esfuerzo social en favor de las comunidades por medio de la intervención de las unidades del Ejército que se encontraban en zonas periféricas del territorio nacional. Para esto se creó, en el 2002, el Centro de Coordinación de Acción Integral (CCAI), cuyo objetivo fue articular los esfuerzos civiles y militares en pro de la recuperación efectiva del territorio. En este equipo participaban 14 entidades del Estado que se reunían para discutir la aplicación de una política social efectiva de manera conjunta e interagencial.

Con el gobierno de Juan Manuel Santos (2010-2018) se determinó, en el 20I I, que el Ministerio de Defensa debía hacer presencia en aquellas zonas olvidadas por el Estado, con un trabajo que incluyera el esfuerzo social e institucional de llevar bienestar, desarrollo y oportunidades a las comunidades afectadas por el conflicto. De esta manera, las Unidades Operativas del Ejército Nacional empezaron a contar con una oficina de Acción Integral, es decir, todas las divisiones, brigadas y batallones tenían personal militar a cargo de las misiones no armadas.

Finalmente, en el 2017, el Gobierno creó el Comando de Apoyo de Acción Integral y Desarrollo (CAAID) del Ejército Nacional, integrado por dos brigadas y ocho batallones, conformados por soldados, suboficiales y oficiales especialistas en esta estrategia (Ejército Nacional, 2018). De esta manera, el esfuerzo no armado en Colombia se 
centralizó al crearse un comando independiente bajo una propia unidad de mando, cuya fortaleza radica en tener la capacidad de concentrar sus esfuerzos únicamente en acciones no armadas.

\section{El Comando de Apoyo de Acción}

\section{Integral y Desarrollo en Colombia}

La misión del CAAID es conducir las operaciones de Apoyo de la Defensa a la Autoridad Civil (ADAC) "en apoyo a las unidades del Ejército Nacional y en coordinación con las instituciones de Estado y de Gobierno, con el fin de generar las condiciones para promover la recuperación social del territorio" (Ejército Nacional, 2018). Su estructura está definida por un comando central ubicado en Bogotá (unidad operativa mayor), dos brigadas (unidades operativas menores), cada una con cuatro batallones (unidades tácticas) (figura I).

La Brigada de Apoyo de Acción Integral y Desarrollo Norte, con puesto de mando en San José de Cúcuta, Norte de Santander, tiene a su cargo los BAAID número I (Costa Caribe), 2 (Santanderes), 7 (Antioquia) y 8 (Casanare y Arauca). Su finalidad es brindarle apoyo a la Primera, Segunda, Séptima y Octava División del Ejército Nacional. Por su parte, la Brigada Sur, con puesto de mando en Cali, Valle del Cauca, tiene como jurisdicción los BAAID número 3 (Valle del Cauca), 4 (Meta), 5 (Cundinamarca) y 6 (Florencia), y tiene como fin lograr la victoria militar, el control territorial y contribuir a la consolidación de la paz en el país.

La importancia de la creación de estas unidades radica en la centralización de los esfuerzos no armados del Ejército. Los soldados que están vinculados a estos batallones son designados de manera exclusiva para esta labor y solo se dedican a desarrollar acciones sociales, pues esta es su misión institucional. Esta medida disminuye el riesgo de que dicho personal sea empleado en labores armadas u operacionales. Además, por medio del programa Fe en Colombia, los BAAID cuentan con profesionales civiles, expertos en temas de comunicaciones, proyectos, historia y otras especialidades, encargados de gestionar la oferta de otras instituciones del Estado con el Ejército Nacional, así como de resolver los problemas sociales en las diferentes zonas del país (Mejía, 2017; Valencia, 2016).

\section{Los batallones de Apoyo de Acción Integral y \\ Desarrollo en Santander y Cundinamarca \\ Características generales}

En términos generales, las acciones integrales que se adelantan en estos batallones se orientan a favorecer a la población más vulnerable de su jurisdicción, reduciendo las capacidades ilícitas de los grupos al margen de la ley, la

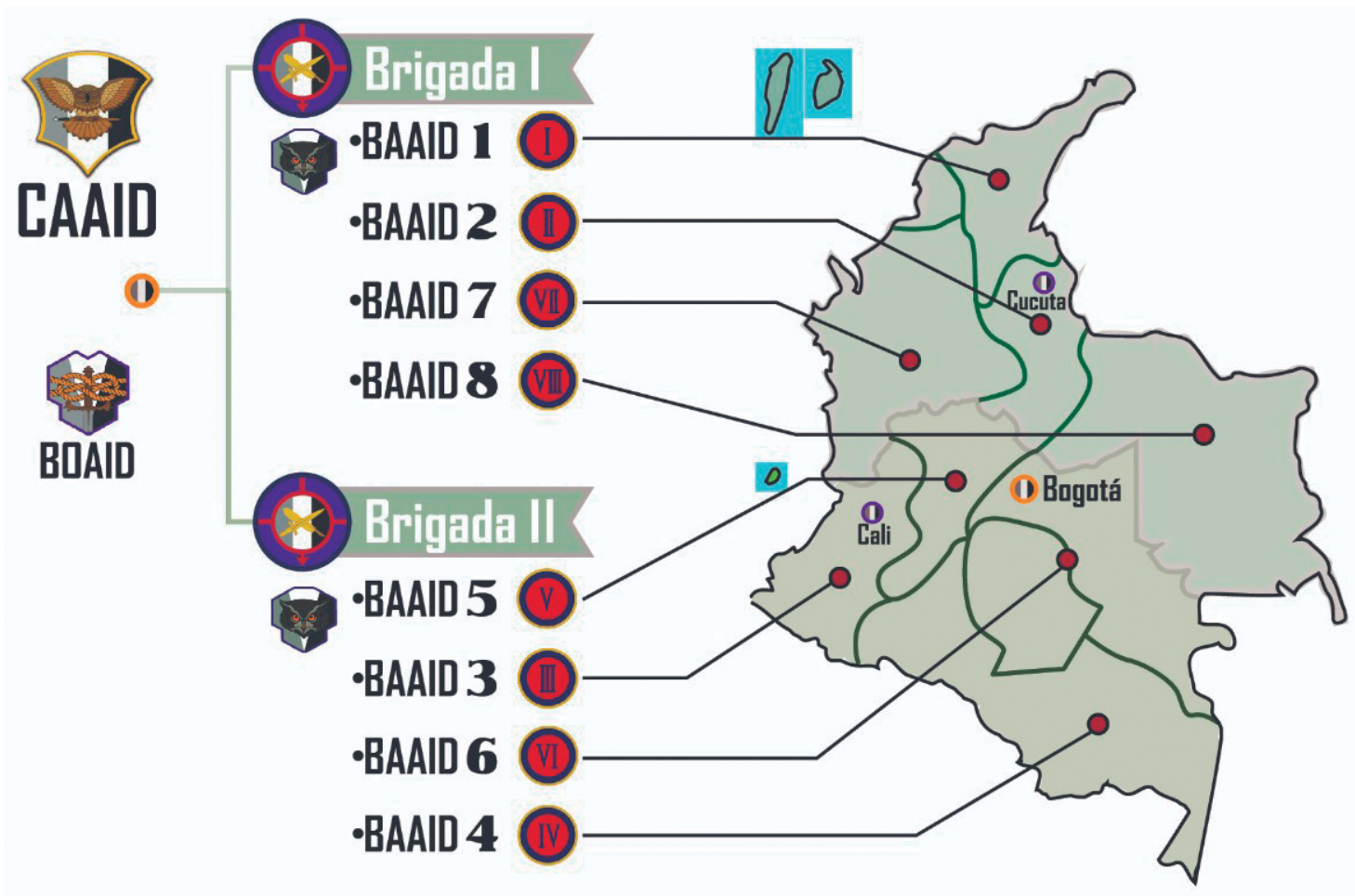

Figura I. Organización territorial del CAAID. Fuente: Ejército Nacional (2017). 
pobreza de las comunidades y recuperando la confianza en la institución, por medio del trabajo articulado entre el Ejército, las instituciones del Estado, el sector privado y los organismos internacionales. Según López (20l8), estas unidades "apoyan el concepto operacional del comandante de división, focalizan las necesidades de la región y revisan cómo estas se pueden abordar desde las capacidades de Acción Integral" (p. I).

Una mirada sobre los BAAID 2 (Santander) y 5 (Cundinamarca) permite deducir que ambas unidades militares tienen una amplia jurisdicción por la cual responder. En el caso del BAAID 2, la jurisdicción corresponde a la Segunda División del Ejército, la cual tiene a su cargo los departamentos de Santander, Norte de Santander, Boyacá, un municipio del oriente de Antioquia, algunos municipios del sur de Bolívar, sur del Cesar y Cundinamarca. La zona a cargo del BAAID 5 es la misma de la Quinta División del Ejército, solo que concentra su esfuerzo en el sur del Tolima, debido a la presencia de disidencias de la FARC y otros grupos armados ilegales que dejaron "fracturas sociales en esta región" (Figura 2).
Al BAAID de Santander lo integran 94 miembros (dos oficiales, diez suboficiales, 73 soldados y nueve profesionales). Por su parte, el BAAID de Cundinamarca está conformado por 120 personas (dos oficiales, 15 suboficiales, 93 soldados y diez profesionales). Los oficiales y suboficiales son los encargados de liderar, en términos organizacionales, las acciones de la estrategia no armada que los soldados ejecutan, mientras que los profesionales son responsables de apoyar la estrategia de Fe en Colombia y promover la gestión de proyectos con instituciones públicas y privadas en beneficio de la población civil.

Según los comandantes de estos batallones, los integrantes fueron seleccionados por su experiencia, puesto que algunos pertenecían a Compañías de Acción Integral, al Grupo Especial de Operaciones Sicológicas u oficinas de Acción Integral.Asimismo, todos fueron capacitados en la Escuela de Relaciones Civiles y Militares en Bogotá, y cada seis meses son reentrenados en el ámbito de acción integral.

En términos presupuestales, se evidencia en ambos batallones la ausencia de partidas destinadas a labores sociales.
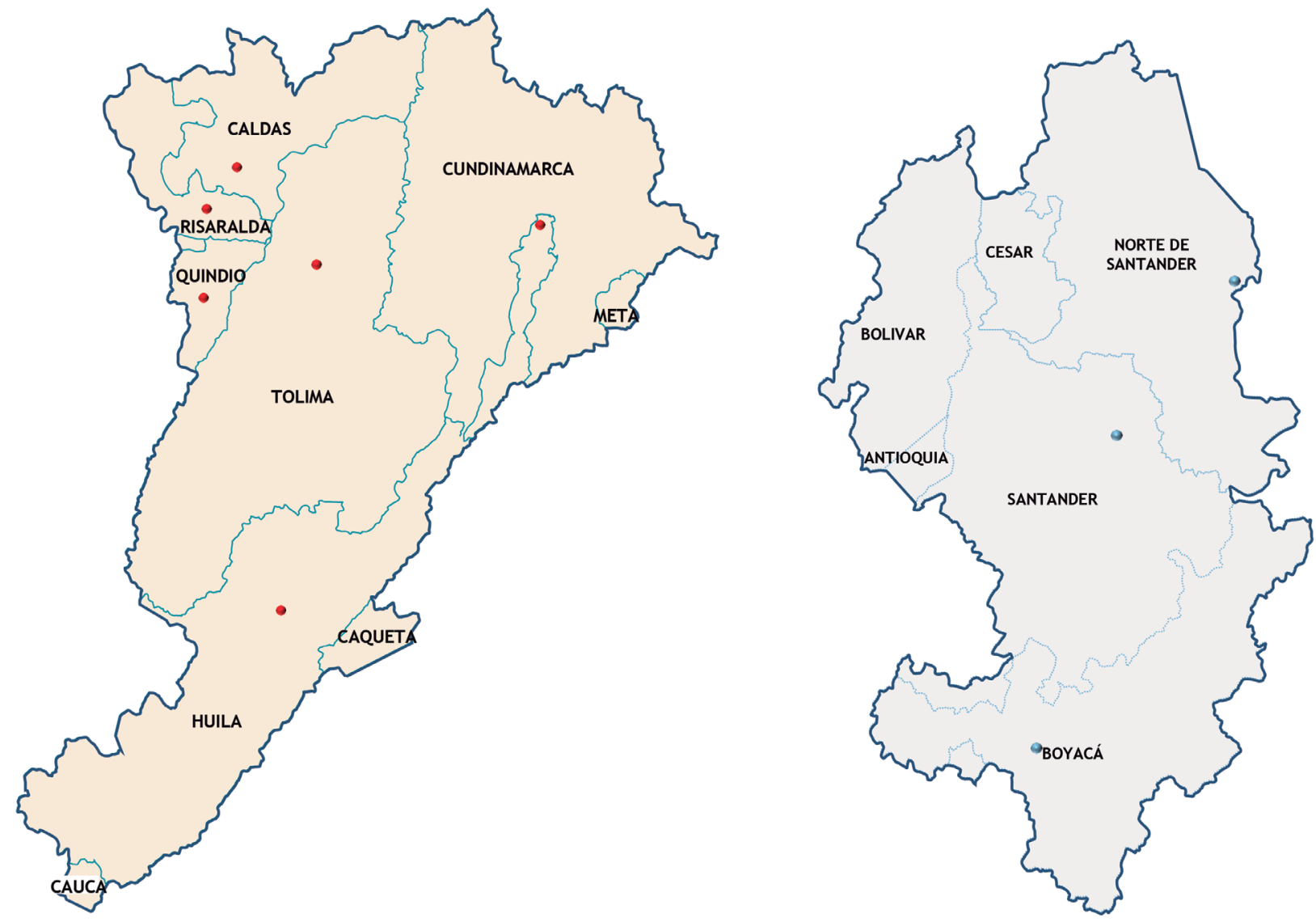

Bucaramanga. Batallón de Acción Integral No. 2 Chaparral. Batallón de Acción Integral No. 5

Figura 2. Jurisdicción BAAID 2 y 5 . Fuente: Ejército Nacional (2017). 
Reciben recursos para gastos de papelería, viáticos y pago del personal profesional. En el BAAID 2, contaron con un presupuesto anual en el 2018 de COP 23606000 , y, según su comandante, los recursos económicos y humanos son insuficientes para cumplir con la misión del Batallón. El comandante del BAAID 5 argumenta que no cuentan con presupuesto porque ellos son articuladores, "lo que nosotros hacemos es ayudarle a la población por medio de la oferta de otras instituciones". A su vez, considera que los recursos nunca serán suficientes, ya que la población siempre tendrán muchas más necesidades.

Entre las líneas de trabajo de los Batallones se destacan cuatro: (I) Infraestructura (mejoramiento de vías, parques infantiles, salones comunales, escuelas, escenarios deportivos y construcción de baterías escolares); (2) Proyectos productivos (agroecológicos, establecimiento de modelos unifamiliares empresariales, ferias de emprendedores e industrialización de cultivos); (3) Medioambiente (estufas ecoeficientes, brigadas de limpieza y siembra de árboles); y (4) Proyección social (ferias, caravanas, exposiciones, actos culturales, brigadas).

En términos generales, las acciones desarrolladas por los integrantes de los estos BAAID son netamente comunitarias y recreativas. No obstante, existe carencia de proyectos o estrategias sostenibles en el tiempo, a excepción de las obras de infraestructura y el trabajo desarrollado con las juntas de acción comunal. En general, son bastante activos en términos lúdicos, pero difícilmente se evidencian estrategias proyectadas a largo plazo (véase la tabla 2).

\section{Aspectos sociodemográficos}

En términos de género, se destaca que el índice de participación femenina en estas unidades del Ejército es reducido, puesto que el $100 \%$ de los encuestados en el BAAID de Santander eran hombres, $y$ en el de Cundinamarca solo el $26 \%$ eran mujeres. Si bien el predominio del género masculino no es una anomalía en el interior de la institución, la participación de las mujeres en este tipo de escenarios sociales puede facilitar el acercamiento del Ejército a la comunidad, lo que se establece como una oportunidad para fortalecer estos batallones.

Ambos BAAID están integrados, principalmente, por personas que se encuentran entre los 20 y los 30 años de edad (Santander 61\% y Cundinamarca al 47\%), seguidas del grupo de $3 \mathrm{I}$ a 40 años (34\% y $43 \%$, respectivamente). Esta información resulta relevante en la implementación de la estrategia no armada, debido a que es un personal con potencial para acumular amplia experiencia en acciones integrales.

Tanto en Santander como en Cundinamarca la mayoría de los encuestados indicaron tener entre 10 y 15 años de servicio militar (BAAID $253 \%$ y BAAID 5 43\%). En menor proporción, se destacan los que han servido a la institución por más de 20 años ( $10 \%$ y $4 \%$, respectivamente). Lo anterior implica que en ambos casos los BAAID cuentan con un número importante de integrantes con amplia trayectoria militar, lo que resulta favorable para la implementación de la estrategia de Acción Integral, en

Tabla 2. Acciones sociales desarrolladas en los BAAID 2 y 5

\section{BAAID Santander}

Actividades de Semana Santa.

Actividades lúdico-recreativas.

Jornadas de salud y lúdico-recreativas.

Jornadas de apoyo y desarrollo humanitario.

Obras sociales en apoyo con alumnos del grado once de los colegios (cumplimiento de horas sociales).

Programa “Jóvenes de Paz" organizado por la Iglesia católica.

Jornadas de apoyo al desarrollo.

Integración lúdica con la población civil.

Acciones de operación psicológica.

Eventos culturales.

Apoyo a población afectada por catástrofes ambientales.

Brigadas de salud.

Circo militar "Risas por la Paz".

Presentaciones del grupo musical del Ejército Nacional.

\section{BAAID Cundinamarca}

Actividades de integración y coordinación con las Juntas de Acción Comunal.

Jornadas ambientales, campañas de siembras y huertas caseras. Jornadas de apoyo al desarrollo.

Capacitaciones comunitarias y obras de infraestructura.

Aplicación de campañas institucionales.

Actividades con diferentes entidades del Gobierno nacional.

Apoyo a la radio comunitaria.

Apoyo a actos culturales y religiosos con el grupo musical.

Fuente: elaboración propia con base en las encuestas aplicadas. 
la medida en que conocen la institución militar y el viraje que ha tenido el papel del Ejército según las necesidades del contexto político. No obstante, también implica un gran reto en torno a la moderación del perfil de acción de aquellos que llevan más de cinco años de servicio y han apoyado unidades militares con vocación opuesta al trabajo comunitario.

Con respecto a la experiencia en labores operacionales, se destaca que en ambos batallones los integrantes de corte militar manifestaron haber participado en acciones armadas. Primó en estas actividades una experiencia acumulada de entre cinco y diez años (48\%), seguidos de aquellos que duraron menos de cinco años $(29 \%)$ y los que llevaban más de diez años ejecutando este tipo de misiones (18\%). Lo anterior ratifica la necesidad de dotar de capacidades técnicas e intelectuales a los integrantes de los BAAID, a fin de que estén en capacidad de intervenir en la población civil y gestionar de manera eficiente la estrategia no armada. Asimismo, es pertinente revisar el perfil de los comandantes, pues ellos, al ser del arma de Infantería, han pertenecido a unidades de combate, han ocupado cargos operacionales, de inteligencia y de manejo de personal, lo que conlleva a redoblar esfuerzos de capacitación en aras de fortalecer el espíritu no armado y comunitario'.

\section{Conocimiento y percepción sobre la Acción Integral del Ejército}

Frente al nivel de conocimiento sobre Acción Integral de los integrantes de los BAAID se evidenció una diferencia significativa, pues en Santander los encuestados indicaron, en su mayoría, contar con un nivel de conocimiento medio sobre el tema (59\%), seguidos de los que eligieron el nivel alto (34\%) y el bajo (6,8 \%). Por su parte, en Cundinamarca, la mayoría argumentó tener alto conocimiento frente al área de acción integral (54\%), seguida del nivel medio $(41 \%)$ y bajo $(4,5 \%)$.

Lo anterior está estrechamente relacionado con que en el Batallón de Cundinamarca el $50 \%$ de los encuestados indicaron que en su formación militar de base habían recibido formación social y comunitaria, mientras que en

I La investigación permitió identificar que, en el caso del comandante del BAAID 2, sus buenos resultados operacionales le permitieron acceder a un curso de formación de dos meses en Acción Integral, lo que le posibilitó liderar el batallón, de manera que fue esa su única experiencia previa en acciones no armadas. Por su parte, el comandante del BAAID 5 contaba con una experiencia más amplia en esta área de acción, pues después de realizar el curso fue durante tres años instructor $y$ ha estado constantemente capacitándose al respecto.
Santander solo el $34 \%$ la recibió. Estos resultados contrastan con lo indicado por los comandantes de los batallones en las entrevistas cuando indicaron que los criterios de vinculación del personal se encuentran asociados a la experiencia de los oficiales, de los suboficiales y de los soldados en esta área de acción.

Los resultados sobre el nivel de conocimiento permiten afirmar que, en general, los integrantes de estos batallones comprenden el papel y la importancia que la misión no armada del Ejército Nacional tiene para el beneficio de la población civil, lo que favorece la implementación de la estrategia de Acción Integral, de acuerdo con lo dispuesto en la norma y los requerimientos que exige la intervención militar sin armas. No obstante, es innegable la necesidad de incrementar la capacitación del 100\% del personal de los BAAID respecto al tema.

Las fortalezas y las debilidades que frente al nivel de conocimiento sobre Acción Integral tiene el personal de los batallones se relaciona estrechamente con la capacitación a la que han podido acceder. Un porcentaje muy significativo de los miembros de ambas unidades han recibido formación en acción integral (86\% en Santander y $95 \%$ en Cundinamarca). Sin embargo, algunos integrantes de estos batallones manifestaron no haber sido capacitados en la estrategia, lo que puede afectar la misión de estas unidades y el sentido de pertenencia de sus miembros.

En términos generales, la formación en Acción Integral que han recibido ha sido impartida por el Ejército Nacional. Los contenidos en los que se han capacitado son diversos y han girado en torno a derechos sociales, culturales $y$ recreativos. No obstante, ninguno de los encuestados indicó haberse capacitado en temas relacionados con la gestión de proyectos, lo que resulta fundamental para la eficiencia de su gestión (véase la tabla 2). Asimismo, se ratificó que la formación previa en Acción Integral no fue un requisito para ser parte de los BAAID, pues solo el $61 \%$ de los integrantes del Batallón Santander y el $50 \%$ de los del Batallón de Cundinamarca realizaron capacitaciones antes de vincularse a esta unidad militar.

En cuanto al tiempo de vinculación del personal en Acción Integral, se destaca que en ambas unidades militares la mayoría de los integrantes acumulan entre uno y tres años de experiencia (39\% en Santander y 59\% en Cundinamarca), lo que es consecuente con el año de creación de estos batallones (2017). El resto de los integrantes 
Tabla 3. Formación en Acción Integral de los integrantes de los BAAID 2 y 5

\begin{tabular}{|c|c|c|c|}
\hline Temas de formación & $\begin{array}{l}\text { Institución } \\
\text { formadora }\end{array}$ & BAAID 2 & BAAID 5 \\
\hline Acción Integral & $\begin{array}{l}\text { Ejército } \\
\text { Nacional y } \\
\text { agencias de } \\
\text { EE. UU. }\end{array}$ & $\mathbf{x}$ & $\mathbf{x}$ \\
\hline $\begin{array}{l}\text { Locución, producción } \\
\text { radial y Fe en Colombia }\end{array}$ & $\begin{array}{l}\text { Ejército } \\
\text { Nacional }\end{array}$ & & $x$ \\
\hline Trabajo comunitario & $\begin{array}{l}\text { Ejército } \\
\text { Nacional }\end{array}$ & $\mathbf{x}$ & \\
\hline $\begin{array}{l}\text { Derechos humanos, } \\
\text { grupos étnicos y música }\end{array}$ & $\begin{array}{c}\text { Casa de la } \\
\text { Cultura San } \\
\text { Martín de Loba }\end{array}$ & $\mathbf{x}$ & \\
\hline $\begin{array}{l}\text { Curso Grupo Especial } \\
\text { de Operaciones } \\
\text { Sicológicas (GEOS) }\end{array}$ & ESMAI & $\mathbf{x}$ & $\mathbf{x}$ \\
\hline $\begin{array}{l}\text { Capacitación en } \\
\text { operaciones psicológicas }\end{array}$ & BAAID & $\mathbf{x}$ & $\mathbf{x}$ \\
\hline $\begin{array}{l}\text { Curso de reentrenamiento } \\
\text { de las Compañías de Acción } \\
\text { Integral (COPAl) }\end{array}$ & BITER \# 5 & $\mathbf{x}$ & $\mathbf{x}$ \\
\hline $\begin{array}{l}\text { Curso de Infancia } \\
\text { y Adolescencia }\end{array}$ & ESMAI e ICBF & $\mathbf{x}$ & $\mathbf{x}$ \\
\hline $\begin{array}{l}\text { Curso de escalonamiento } \\
\text { en el arma Acción Integral }\end{array}$ & $\begin{array}{l}\text { Ejército } \\
\text { Nacional }\end{array}$ & & $x$ \\
\hline $\begin{array}{l}\text { Preparación en } \\
\text { temas sociales }\end{array}$ & Universidades & $\mathbf{x}$ & $\mathbf{x}$ \\
\hline
\end{tabular}

Fuente: elaboración propia con base en las encuestas aplicadas.

tienen más de tres años de experiencia, lo que repercute de manera positiva en las acciones comunitarias y el relacionamiento estratégico, pues existen bases que desde la práctica permiten lograrlo.

La experiencia sumada al conocimiento le aporta al militar que se desempeña en Acción Integral un valor agregado y diferencial en el marco de su nueva misión institucional, al contar con una cosmovisión frente a las funciones de un militar que no defiende los intereses de la población por medio de las armas, sino a través de acciones sociales que contribuyen al bienestar, el desarrollo y la superación del conflicto.

Percepción frente a la estrategia de Acción Integral

Con respecto a la percepción que los integrantes de los BAAID tienen sobre la forma en que se reconoce su labor por parte de otras unidades del Ejército Nacional y de la sociedad civil, se destaca tanto en Santander como en Cundinamarca que los militares vinculados a otras áreas de acción no valoran de manera significativa la misión no armada y la consideran de importancia secundaria para la institución:
La mayoría de generales, coroneles, tenientes y soldados no comprenden la importancia de la Acción Integral, se concentran en obtener otro tipo de resultados que les permiten adquirir medallas y ascender en la institución, por eso no la apoyan como deberían. (Comunicación personal, 10/10/2018, JQB)

Por el contrario, resaltan la valoración positiva, el agradecimiento y el alto reconocimiento que reciben por parte de la población civil, los principales destinatarios y beneficiarios de sus acciones sociales:

Lo que hacemos tiene gran acogida por parte de la comunidad. Siempre manifiestan su agradecimiento con los soldados y las personas que integran el batallón. La gente recibe bien todo lo que hacemos nosotros. Viven encantados porque hay zonas donde ese tipo de apoyos no se habían visto. (Comunicación personal, 10/10/2018, JQB)

La acogida y la gratitud de la comunidad tiene mucho que ver con las razones que los integrantes de estos batallones esgrimen para argumentar su alto nivel de satisfacción frente a la labor desempeñada en estas unidades. En especial, destacan la alegría de los niños y el apoyo a las personas más vulnerables como los aspectos que más les gusta de su misión. De esta manera, identifican su trabajo como un canal cuyo propósito es llevar bienestar social y desarrollo a las comunidades (véase la tabla 4).

Asimismo, reconocen que son muchas las experiencias positivas que les ha dejado el trabajo social y comunitario en cada uno de los batallones. Esas experiencias están en estrecha armonía con la respuesta afectuosa de la comunidad, eje que da sentido a su accionar y compensa los sacrificios del ejercicio militar (véase la tabla 5).

Para el comandante del BAAID 5, los batallones de Acción Integral son muy importantes porque le permiten al Ejército Nacional "tenderle una mano colaborativa a la población civil” (comunicación personal, I5/I0/20/8, MBG). Considera que van por muy buen camino $y$, a pesar de que deben mejorar el relacionamiento con otras instituciones, afirma que con mucho esfuerzo lo están logrando. Ejemplo de ello son los proyectos productivos que están realizando en temas agrícolas y piscícolas, las actividades culturales y artísticas, así como las obras de infraestructura.

El grado de satisfacción del personal de los BAAID es consecuente con su deseo de permanecer en estas unidades. El $83 \%$ de los encuestados del Batallón de Santander 
Tabla 4. Razones por las que les gusta su labor en Acción Integral

\section{BAAID Santander}

Tener la oportunidad de llegar a las poblaciones más apartadas y llevarle sonrisas a los niños.

Servirle a la población civil respecto a lo que requieren.

El afecto que la población siente por los soldados.

Es grato tener que trabajar por los niños y compartir con la población menos beneficiada por el Estado.

Ver feliz a la comunidad que no han tenido una jornada médica o actividad recreativa. Tener la oportunidad de llegar a donde nadie lo ha hecho y de ayudar a la comunidad más vulnerable.

Ver la sonrisa de la población civil y la de las propias tropas al finalizar una actividad.

La satisfacción de llegar a un pueblo o a una vereda y robarle una sonrisa a todos nuestros niños.

Me gusta hacer sonreír a los niños y a los adultos mayores, ver satisfecha a la población civil y en especial a los niños.

Lo que más me gusta es mirar el alcance que tenemos para contrarrestar el accionar del enemigo y ganarnos el aprecio de la población civil.

Disfruto ver a la gente feliz, ellos se van agradecidos con las jornadas.

Me gusta ayudar al desarrollo de la población.

Fuente: elaboración propia con base en las encuestas aplicadas.

Tabla 5. Experiencias positivas de la Acción Integral

\section{BAAID Santander}

\section{BAAID Cundinamarca}

Saber que el trabajo que haces es recompensado con unas "gracias", una sonrisa, un abrazo de la comunidad. Eso es felicidad.

Servirle a las personas y brindar lo mejor de mí.

Apoyar a las comunidades más necesitadas.

Ver cómo la comunidad se acerca más al Ejército y se beneficia de las actividades que desarrollamos en mi batallón.

Me gusta servir a la comunidad, llegar a las zonas apartadas, ver la felicidad de la gente cuando el Ejército Nacional y el programa Fe en Colombia llega, es algo que no tiene precio.

La integración de todo el aparato estatal con el fin de ayudar a satisfacer las necesidades insatisfechas de la comunidad.

La alegría de las personas cuando son apoyadas.

Me gusta contribuir a mitigar los efectos de la violencia, la desigualdad o vulnerabilidad a través de la Acción Integral.

La satisfacción de hacer algo, de sentir que se está dejando huella.

Me gusta que estamos apoyando el progreso de las regiones y es gratificante ver cómo la comunidad disfruta de nuestro trabajo.

La satisfacción de la felicidad reflejada en las comunidades que visitamos. Llevamos diversión y desarrollo.

\section{BAAID Cundinamarca}

Con información que nos dieron evitamos que se llevaran a unas niñas a La alegría de las personas después de las actividades.

la guerra y que no cayera la tropa.

La desmovilización de los grupos armados ilegales.

En las actividades se nota la satisfacción por parte de la población beneficiada. La sonrisa de los niños recibiendo un globo.

Ver como una población nos da acogida amablemente, agradeciendo nuestra labor.

En realidad, son muchas. Cuando estuve en resguardos indígenas, había niños que nunca vieron un payaso y como yo fui de payaso, fue muy bonito porque todos me querían abrazar; $y$ en las jornadas de apoyo al desarrollo los abuelitos son los más agradecidos, siempre esos abrazos y esas bendiciones que nos dan.

Las lágrimas de agradecimiento y felicidad de un líder de una población a la cual llevamos proyectos productivos para el desarrollo de esta región. Tengo muchos recuerdos, pero en particular uno que nos sucedió en el municipio Las Mercedes (Norte de Santander), donde terminábamos con el ciclo de las actividades y con lágrimas en los ojos los niños nos pedían que no nos fuéramos, que nos quedáramos más tiempo. Ese es el momento en que uno personalmente dice que está haciendo las cosas bien.

Una de mis experiencias más recordadas fue trabajar con la comunidad indígena.

Cuando se han realizado jornadas con poblaciones marginadas, las cuales quedan muy agradecidas. También en los colegios, universidades y ancianatos nos hacen sentir que somos importantes para ellos.

La gratitud de la población apoyada, las experiencias que comparten en el marco de actividades como Cátedra de Paz o recopilación de testimonios de memoria histórica.

Llevamos felicidad a los soldados, niños y ancianos.

Todas, me llevo todas las experiencias del mundo siempre positivas. Amo la Acción Integral, amo mi trabajo dentro del batallón.

Es incalculable medir la alegría de los niños cuando ven cómo hacemos trabajos que los benefician. Ver cómo personas que eran 100\% farianas trabajan de la mano con nosotros y se hace una reconversión de su ideología.

En Cartagena del Chairá. Percibir como un pueblo de Colombia con más de cien años de fundado jamás había tenido presencia directa de entidades del Estado y jamás había celebrado unas ferias y fiestas ni nada por el estilo. El calor humano, la acogida y la admiración valen más que mil medallas.

Los agradecimientos de la población al final de cada actividad.

Me ha traído un sin número de experiencias maravillosas que me han servido para ser mejor como persona, a nivel familiar y laboral. El trabajo con los niños, comunidades indígenas como La Guajira y poblaciones vulnerables marcan de por vida.

Son tantas que en realidad pienso en las veces que he visto sonreír a más de una persona y dar las gracias, es lo máximo.

La alegría reflejada en niños y abuelos.

El agradecimiento sincero de la población y su confianza puesta en el Ejército.

Fuente: elaboración propia con base en las encuestas aplicadas.

y el $80 \%$ de Cundinamarca manifestaron no querer trabajar en una unidad diferente a la de Acción Integral. El sentido de pertenencia que expresan facilita la gestión y la organización de las acciones sociales y, sin duda, impacta a la comunidad de manera favorable, con el trabajo realizado con gusto y convicción.
En el caso de los que manifestaron deseo de ser trasladados a otras unidades militares se desatacan argumentos que enfatizan en la precariedad con la que operan los BAAID debido a la limitación de recursos económicos y humanos, los requerimientos de capacitación, la necesidad de invertir recursos personales para ejecutar algunas 
actividades, la impotencia de no poder hacer más por la comunidad, la distancia de sus núcleos familiares y la falta de apoyo de los comandantes militares para el buen desarrollo de sus funciones.

Todas estas razones hacen parte de conjunto de aspectos que los encuestados indicaron no les gusta de su labor (véase la tabla 6).

En ambas unidades los militares señalaron que en los batallones se cuenta con recursos humanos, pero carecen de medios técnicos y financieros. Con relación a lo anterior, el comandante del BAAID de Santander considera que entre las principales dificultades se destaca la falta de asignación de recursos y medios que les permita ser autosuficientes, "sin tener que estarle pidiendo combustible - vehículos a otros batallones" (comunicación personal, 15/I0/20I8); los conflictos entre los mismos oficiales del Ejército Nacional que quieren tener el poder sobre esta unidad; $y$ la ausencia de medios para realizar las actividades y elevar el impacto."Por ejemplo, en este momento la carpa del circo militar está totalmente dañada y no hay de dónde obtener los recursos para restaurarla" (comunicación personal, I5/10/20I8). Por su parte, el comandante del BAAID 5 sostiene que, al ser una unidad nueva, los recursos apenas están ingresando. Sin embargo, afirma que sí requieren más personal.
En la tabla 7 se describen algunas consideraciones hechas por los encuestados respecto a las principales necesidades que enfrentan.

Finalmente, desde la percepción de los miembros de ambos batallones, entre las alternativas para fortalecer la estrategia de Acción Integral del Ejército en Colombia se encuentra la asignación presupuestal, el reconocimiento por parte de otros mandos militares, el apoyo logístico y las capacitaciones. A fin de hacer frente al limitado presupuesto de los batallones resulta indispensable fortalecer las competencias de los integrantes de estas unidades en habilidades blandas que les permitan gestar articulaciones con otros actores institucionales que sí cuenten con el presupuesto para la ejecución de proyectos comunitarios. Esto, además, impide la debida participación de los BAAID en escenarios de articulación asociativa con otras organizaciones.

\section{Conclusiones}

En Colombia, durante los últimos años se ha avanzado en la implementación de la estrategia no armada por parte del Ejército Nacional a través del trabajo social y comunitario en aras de garantizar el bienestar y el desarrollo social. La consolidación de la estretagía ha estado relacionada con la creación, en el 2017, del Comando de Apoyo

Tabla 6. Razones por las que no les gusta su labor en Acción Integral

\section{BAAID 2}

\section{BAAID 5}

No me gusta que hay personas de la institución que aún ven la Acción Integral como el payaso, el de los cumpleaños, el de las novenas, el de la misa, no ven más allá.

no disponemos de buenas herramientas para trabajar y hacer las cosas mejor.

Que luego de que los soldados son capacitados, los sacan de Acción Integral.

No me gusta la falta de apoyo del Ejército y otras unidades para poder cumplir con efectividad la misión de ayudar a la población vulnerable.

No me gusta que hay comandantes de unidades que no nos brindan el apoyo para poder realizar nuestro trabajo.

No me gusta que el batallón no es autónomo para el desempeño de la misión al carecer de vehículos y material de trabajo en buen estado.

No me gusta que falta capacitación y material para trabajar.

La falta de continuidad en el trabajo con la comunidad que está en los municipios más afectados por el conflicto armado.

No me gusta que no reconozcan nuestro trabajo.

Algunos mandos son reacios a la implementación de la Acción Integral como la forma de ganar la población civil.

Falta de recursos y seguridad para realizar las actividades.

No tenemos los medios, ni el material, ni el transporte.

Mayor seguridad para cumplir nuestra misión.

Falta material didáctico y logístico para el desarrollo de las actividades de Acción Integral.

Dificultades como alimentación, hospedaje, transporte y seguridad.

Los batallones de Acción no tienen el presupuesto y los soldados están trabaNo me gusta que algunos militares tienen un trato poco amable con los civiles y militares que pertenecemos al área de Acción Integral. De hecho, hay militares que recién recibieron arma, y no se ponen el distintivo porque hay militares de mayor rango que demeritan el trabajo de quienes pertenecemos a un BAAID.

Falta de recursos para los batallones para desempeñar las labores de Acción Integral.

No me gusta el poco apoyo que brindan algunos comandantes de las unidades militares al tema de Acción Integral.

Falta de personal y apoyo financiero en las unidades.

No hay material suficiente en los batallones para trabajar.

No contamos con vehículos para movilizarnos.

El poco apoyo de algunas instituciones.

Que muchos militares ven la Acción Integral como los payasos y no como un mecanismo para ayudar acercarse a la comunidad.

Algunos superiores no ven la Acción Integral de forma estratégica.

La necesidad de vehículos y la asignación de partidas para comprar el material para llevar a cabo la misión.

La parte logística que es de bastante complejidad porque como Batallón no tenemos vehículos propios y dependemos de los de otras unidades. Segundo, el acceso a los sitios tan apartados donde muchas veces no entran los vehículos, sino que toca a lomo de mula o caminando. jando con un material de hace diez años, obsoleto.

Fuente: elaboración propia con base en las encuestas aplicadas. 
Tabla 7. Principales necesidades que enfrentan los BAAID

Falta de implementos como trajes, pinturas, globos, sonido, micrófonos e inflables.

El apoyo o las partidas que se dan para ciertas actividades. Son muy pocas. Falta de recursos para arreglar el material.

No tenemos materiales para trabajar.

Falta más apoyo logístico.

Falta material de pinturita, saltarín, teatro.

Lo humano está, pero en lo económico y en lo técnico no hay absolutamente nada.

La Acción Integral requiere de un presupuesto mayor al de mi sueldo.

Falta apoyo en el mantenimiento de las herramientas.

Ningún comandante invierte los recursos en Acción Integral, y al batallón no le ha llegado ninguna partida como tal.

Faltan recursos económicos para el mantenimiento de los instrumentos y de los implementos de los artistas.

Fuente: elaboración propia con base en las encuestas aplicadas.

Tabla 8. Alternativas para fortalecer la estrategia de Acción Integral
BAAID Cundinamarca

Creando partidas y compra de material técnico para llevar a cabo la misión y vehículos, capacitación y personal, ya que los BAAID no cuentan con personal para llevar a cabo la misión.

Podríamos fortalecerla con más apoyos económicos y humanos en las unidades de Acción Integral y Desarrollo.

Dándonos más lazos de amistad y trabajo en común.

Mayor apoyo económico de parte del Comando del Ejército a los BAAID

y los equipos de Fe en Colombia. Creación de escenarios de interacción con cooperantes internacionales y sujetos de responsabilidad social empresarial.

Dando a conocer estas acciones de las que la gente desconoce y motivar a las personas a hacer voluntariados, donaciones y ayudar.

Con los círculos de calidad o trabajo más vinculante en la toma de ideas con el personal de más experiencia de los grupos musicales y de Acción Integral.

Con un mayor personal en las unidades militares para tener una mayor cobertura con nuestras actividades.

Trabajando así como venimos articulados con los diferentes entes del Estado.

Vinculando empresas privadas para que hagan parte y sean activos en toda ocasión.

El acompañamiento civil es muy importante, hablo desde lo que hago. Cada batallón debe tener un vivero. Nosotros hemos entregado a la comunidad 15000 plantas forestales y 5000 hortalizas. Todo para la protección de fuentes hídricas y la seguridad alimentaria de las poblaciones más vulnerables.

En la Escuela Superior de Guerra sería muy importante que dieran una muy buena capacitación de Acción Integral y Desarrollo a los nuevos señores teniente coroneles y generales. En lo posible lograr que los cuadros tengan un segundo idioma. A los agregados militares deberían darles tareas de Acción Integral, por ejemplo, que al término de su agregación regresen con convenios de cooperación en beneficio de la institución o de las comunidades.

conocimiento y les guste la Acción Integral.

Material para el desarrollo de actividades, para entregar a la población civil y más compromiso de las otras instituciones.

Capacitando más el personal que integran estos batallones y que los más efectiva.

Impulsando programas eficaces que brinden una seria ayuda: esto aumenta la credibilidad.

Más recursos para los batallones de Acción Integral, así se fortalece esta estrategia.

Adelantando más programas por parte del Estado en las diferentes áreas. Fortalecer los lazos de amistad con los entes territoriales. Personal idóneo para preparar y fortalecer el conocimiento y así transmitirlo.

Fuente: elaboración propia con base en las encuestas aplicadas.

de Acción Integral y Desarrollo (CAAID), integrado por ocho batallones de Apoyo de Acción Integral y Desarrollo (BAAID), desplegados por todo el territorio nacional.
La investigación adelantada sobre los BAAID de Santander y Cundinamarca permitió identificar una serie de debilidades, oportunidades, fortalezas y amenazas que enfrentan 
estos batallones y que, por ser estructurales, podrían extrapolarse a las demás unidades que integran el CAAID.

Entre las debilidades de los batallones se destaca que no cuentan con recursos ténicos ni financieros para el desarrollo de actividades sociales y comunitarias. Las partidas económicas asignadas son para el sostenimiento administrativo y el pago de nómina de los profesionales. Por tanto, enfrentan una limitación logística muy alta, al no ser autónomos y estar supeditados al apoyo de otras unidades militares y a la articulación con otras entidades del Estado. Estas unidades, al depender de los recursos de otras instituciones y organizaciones, no tienen la autonomía suficiente para beneficiar a las poblaciones vulnerables.

Esta dependencia resulta compleja en un contexto caracterizado por la valoración baja que sobre la Acción Integral presentan algunos militares vinculados a otro tipo de acciones en el Ejército. La percepción de los integrantes de los BAAID sobre la falta de reconocimiento a su labor por parte de la institución es una debilidad que entorpece su capacidad lógistica e invita ha fortalecer la conciencia militar sobre los efectos positivos de este tipo de intervenciones no armadas.

Adicionalmente, se establece como una debilidad el hecho de que no todos los integrantes de los BAAID cuenten con formación en Acción Integral. La capacitación es un aspecto a mejorar y debería ser requisito previo para el ingreso tanto del personal militar como del civil a estas unidades. Capacitar en habilidades blandas para el relacionamiento interinstitucional es una necesidad en los batallones, en cuanto estrategia para enfrentar las limitaciones presupuestales.

En torno a las oportunidades identificadas en los batallones de Acción Integral se encuentra el nivel de conocimiento sobre la estrategia no armada que predomina en los integrantes de estos batallones, así como el alto grado de satisfacción que les genera la labor desempeñada en estas unidades. A esto se le suma la integración de un equipo profesional que los apoya y los asesora en esta labor y el reentrenamiento recibido cada seis meses para actualizarlos en derechos humanos. Estos elementos resultan claves a fin de garantizar el éxito de la estrategia.

Entre las princpales fortalezas que fue posible identificar se destaca el afecto, el apoyo y el agradecimiento de la población civil beneficiada con la intervención comunitaria por parte del Ejército, lo que ratifica la funcionalidad de este tipo de esfuerzos institucionales. Asimismo, se encuentra la oferta estatal de servicios, la cual puede potenciar su impacto por medio de la articulación interinstitucional. Al respecto, valdría la pena vincular de manera más significativa a la academía, específicamente en la transferencia de conocimiento.

Finalmente, en cuanto a las amenazas que se identificaron se encuentran aspectos burocráticos del devenir público y privado que retrasan el proceso de artículación, así como las características topográficas de las jurisdicciones en la que operan los batallones, las cuales dificultan la posibilidad de hacer presencia en los lugares del territorio que tradicionalmente han estado más rezagados de la presencia estatal.

\section{Referencias}

Cabrera, F. (2005). Relaciones civiles y militares durante el periodo comprendido entre 1994-2002 y su incidencia en la seguridad nacional (tesis de maestría). Universidad Javeriana, Bogotá. Recuperado de https://repository.javeriana.edu.co/handle/I0554/265

CGFM (Comando General de las Fuerzas Militares). (2017). Manual de acción integral conjunta. Bogotá: Imprenta de las Fuerzas Militares.

Ejército Nacional. (20I8). Ejército Nacional, Patria, Honor y Lealtad. Recuperado de https://www.ejercito.mil.co/conozcanos/mision_vision_362168

Guevara, R. (2016). El estado del arte en la investigación: ¡análisis de los conocimientos acumulados o indagación por nuevos sentidos? Revista Folios, I65-179.

Guevara, D. A., Silva, D. F., \& Villegas, O. A. (2018). Las relaciones entre memoria histórica militar y educación. El caso de las Fuerzas Militares de Colombia en el posacuerdo. Reflexión Política, 20(39), 49-64. https://doi. org/I0.29375/0I24078I.3294

López, H. (2018). Acción integral se fortalece para una Colombia en posconflicto. Revista Militar Diálogo las Américas. Recuperado de https://dialogo-americas.com/es/articles/ accion-integral-se-fortalece-para-una-colombia-en-posconflicto/

Mejía, A. (2017). Batallón de acción integral y desarrollo. Ejército Nacional.

Muñoz, N. (20I I). El estudio exploratorio. Mi aproximación al mundo de la investigación cualitativa. Revista Investigación y Educación en Enfermería, 29(3), 492-499. 
Pereira, Z. (20II). Los diseños de método mixto en la investigación en educación: una experiencia concreta. Revista Electrónica Educare, XI(I), I5-29.

Silva. M. (20/4). La acción integral como una estrategia efectiva hacia la consolidación de la seguridad y la defensa nacional (trabajo de grado). Universidad Militar Nueva Granada, Bogotá. Recuperado de https://repository.unimilitar.edu. co/handle// 0654/I3730
Suárez, J. (2010). Acción Integral 2006-2009. Bogotá: Universidad Javeriana.

Valencia, Á. (2006). Acción Integral: una historia de ayuda y gestión. Revista "Fuerzas Armadas", 198(20), 15-32. 